\title{
The 8th ACM International Conference on PErvasive Technologies Related to Assistive Environments
}

\section{PETRA 2015}

\section{Conference Program}

July 1-3, 2015

Corfu, Greece

Organized by

The University of Texas at Arlington, Arlington, Texas, USA

With sponsorship from:

The National Science Foundation (NSF), USA and the following:

College of Engineering, University of Texas at Arlington, USA

Computer Science and Engineering Department, UTA, USA

HERACLEIA Human Centered Computing Laboratory at UTA, USA DEMOKRITOS- National Center for Scientific Research (NCSR), GREECE University of Piraeus (Department of Digital Systems), GREECE

The Ionian University, GREECE

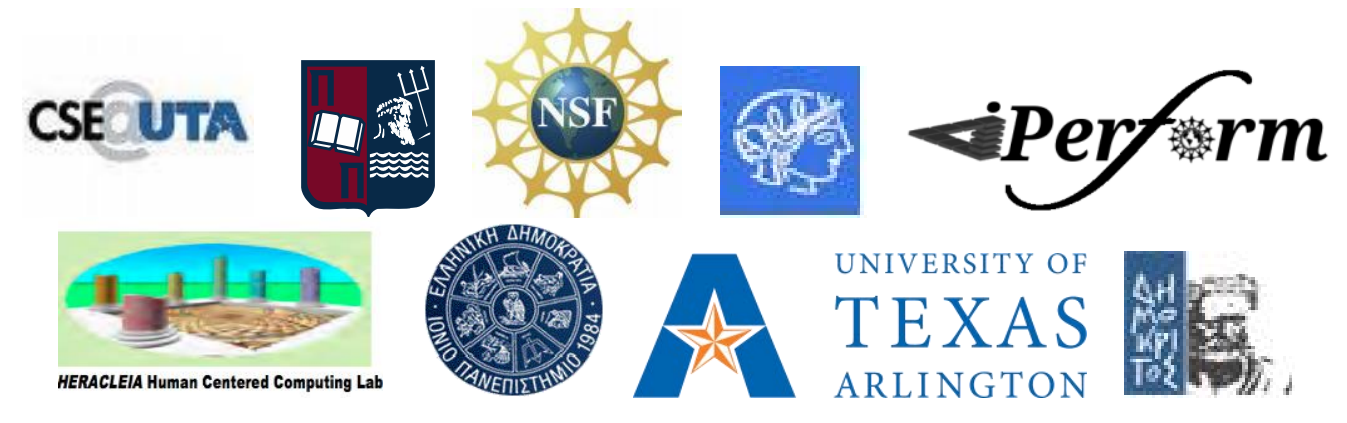

\section{Conference Proceedings}

$\underline{\text { ACM - Association for Computing Machinery }}$

Digital Library Proceedings 
Proceedings of PETRA 2015

The 8th International Conference on PErvasive Technologies Related to Assistive Environments 2015, Corfu, Greece (PETRA '15)

Conference Chair: Dr. Fillia Makedon

Program Chairs: Gian-Luca Mariottini, Oliver Korn, Illias Maglogiannis, Vangelis Metsis Sponsor(s):

- Computer Science \& Engineering Department, UTA (cse.uta.edu)

- The Fulbright Foundation

- The National Center for Scientific Research (NCSR)- DEMOKRITOS, Greece

- The HERACLEIA Human Centered Computing Laboratory at UTA (heracleia.uta.edu)

- The iPerform Center on Assistive Technologies to Enhance Human Performance 


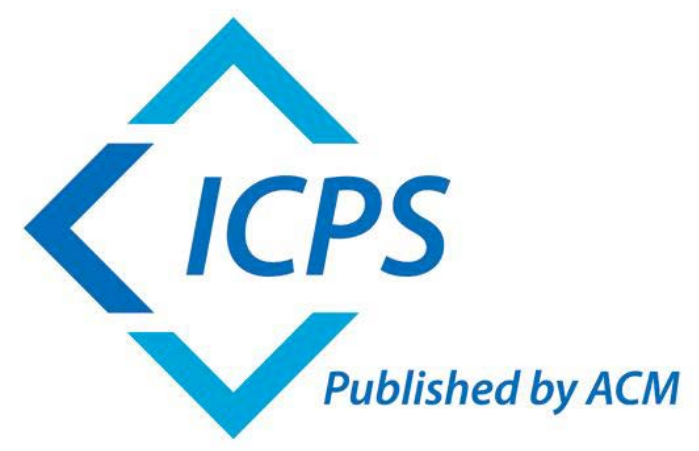

The Association for Computing Machinery

2 Penn Plaza, Suite 701

New York New York 10121-0701

ACM COPYRIGHT NOTICE. Copyright $\odot 2015$ by the Association for Computing Machinery, Inc. Permission to make digital or hard copies of part or all of this work for personal or classroom use is granted without fee provided that copies are not made or distributed for profit or commercial advantage and that copies bear this notice and the full citation on the first page. Copyrights for components of this work owned by others than ACM must be honored. Abstracting with credit is permitted. To copy otherwise, to republish, to post on servers, or to redistribute to lists, requires prior specific permission and/or a fee. Request permissions from Publications Dept., ACM, Inc., fax +1 (212) 869-0481, or permissions@acm.org

For other copying of articles that carry a code at the bottom of the first or last page, copying is permitted provided that the per-copy fee indicated in the code is paid through the Copyright Clearance Center, 222 Rosewood Drive, Danvers, MA 01923, $+1-978-750-8400,+1-978-750-4470$ (fax).

Notice to Past Authors of ACM-Published Articles

ACM intends to create a complete electronic archive of all articles and/or other material previously published by ACM. If you have written a work that was previously published by ACM in any journal or conference proceedings prior to 1978, or any SIG Newsletter at any time, and you do NOT want this work to appear in the ACM Digital Library, please inform permissions@acm.org, stating the title of the work, the author(s), and where and when published.

ACM ISBN: 978-1-4503-3452-5 


\section{Dear Colleagues,}

Welcome to the 8th ACM International Conference on PErvasive Technologies Related to Assistive Environments, July 1-3 2015 in Corfu, Greece. We wish to acknowledge support from the National Science Foundation (NSF) that has awarded us a Doctoral Consortium grant to enable US-based graduate student authors to travel and present their results to the conference. Support was also provided by NSF's Computer Research Infrastructure (CRI) program in order to conduct a planning workshop on developing SMART-MOVE, a multimodal human activity recognition repository, that takes place on the third day of the conference. We also wish to acknowledge support by the Dean of the College of Engineering at the University of Texas Arlington (UTA) for travel grants offered to several UTA student authors.

The PETRA Conference brings together researchers from diverse disciplines who use pervasive computing technologies to improve the quality of life and enhance human performance. Results presented include not only basic research in computer vision, machine learning, data mining and big data, but also engineering applications and software solutions that improve, for example, vocational safety and productivity, physical or mental rehabilitation, better drug treatment, and other areas. The conference addresses the needs of both healthy individuals, as well as those with special needs, such as providing safe in-home care for the elderly, or for persons with Dementia, Alzheimer's, Parkinson's, chronic arthritis, or other conditions. PETRA also addresses the growing need of people living and working in an increasingly crowded and complex world who require inclusiveness and connectivity with the rest of the world. In summary, PETRA's novel computer-based methods and tools can help people build, stone by stone (PETRA means stone in Greek), new ways of life to survive in an increasingly challenging world. Best Paper Awards will be presented at the end of the conference for Best Technical Paper, Best Paper for Novelty, and Best Student Paper

\section{Invited Speakers}

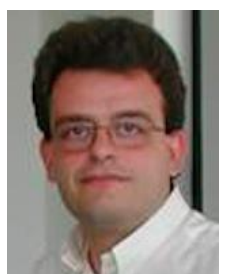

Antonis Argyros is a Professor of Computer Science at the Computer Science Department (CSD), University of Crete (UoC) and a researcher at the Institute of Computer Science (ICS), Foundation for Research and TechnologyHellas (FORTH) in Heraklion, Crete, Greece. He received his B.Sc. (1989) M.Sc. (1992) and Ph.D. (1996) degrees from CSD-UoC. He has been a postdoctoral fellow at the Computational Vision and Active Perception Laboratory (CVAP) at the Royal Institute of Technology (KTH), Stockholm, Sweden. Since 1999, as a member of the Computational Vision and Robotics Laboratory (CVRL) of ICS-FORTH, he has been involved in many RTD projects in computer vision, image analysis and robotics. His research interests fall in the areas of computer vision with emphasis on tracking, human gesture and posture recognition, 3D reconstruction and omnidirectional vision. He is also interested in applications of computer vision in the fields of robotics and smart environments. In these topics, he has published more than 120 papers in peer reviewed scientific journals, conferences and workshops. Antonis Argyros is an area editor for the Computer Vision and Image Understanding Journal (CVIU) and a member of the Editorial Board of the IET Image Processing Journal. He has served as one of the General Chairs of the 11th European Conference in Computer Vision (ECCV'2010) as well as in the organizing and program committees of several vision, graphics and robotics conferences and workshops. He is also a faculty member of the Brain and Mind interdisciplinary graduate program and a member of the Strategy Task Group of the European Consortium for Informatics and Mathematics (ERCIM).

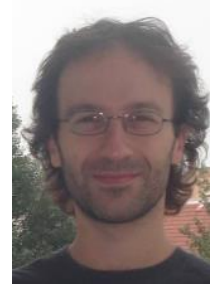

Vassilis Athitsos is Associate Professor in the Department of Computer Science and Engineering (CSE) at the University of Texas at Arlington (UTA), Texas. He received the BS degree in mathematics from the University of Chicago in 1995, the MS degree in computer science from the University of Chicago in 1997, and the Ph.D. degree in computer science from Boston University in 2006. In 2005-2006 he worked as a researcher at Siemens Corporate Research, developing methods for database-guided medical image analysis. In 2006-2007 he was a postdoctoral research associate at the Computer Science department at Boston University. Since August 2007 he is a faculty member at UTA. His research interests include computer vision, machine learning, and data mining. His recent work has focused on gesture and sign language recognition, detection and tracking of humans using computer vision, efficient similarity-based retrieval in multimedia databases, and shape modeling and detection. His research has been supported by the National Science Foundation, including an NSF CAREER award.

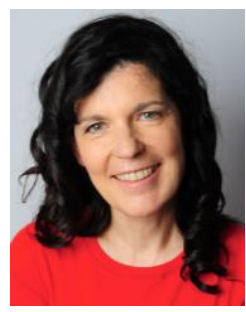

Elisabeth André is a Full Professor of Computer Science at Augsburg University in Germany, and Chair of the Research Unit Human-Centered Multimedia. She received her Diploma and Doctoral Degrees in Computer Science from Saarland University. Elisabeth André has a long track record in multimodal human-machine interaction, embodied conversational agents, affective computing andsocial signal processing. She is on the editorial board of various renowned international journals, such as IEEE Transactions on Affective Computing (TAC), Journal of Autonomous Agents and Multi-Agent Systems (JAAMAS), ACM Transactions on Intelligent Interactive Systems (TIIS) and AI Communications. In 2007, Elisabeth André was nominated Fellow of the Alcatel-Lucent Foundation for Communications Research. In 2010, she was elected a member of the prestigious German Academy of Sciences Leopoldina, the Academy of Europe and AcademiaNet. She is an ECCAI Fellow (European Coordinating Committee for Artificial Intelligence). 


\begin{tabular}{|c|c|c|}
\hline & Room 1 & Room 2 \\
\hline $\begin{array}{l}09: 00- \\
9: 40\end{array}$ & $\begin{array}{r}\text { Confer } \\
\text { Fillia Makedon, Conference } \\
\text { Ilias Maglogiannis, University of Piraeus, Departme }\end{array}$ & $\begin{array}{l}\text { ence Opening } \\
\text { Chair, University of Texas Arlington } \\
\text { tt of Digital Systems, Greece (Program Committee Co-Chair) }\end{array}$ \\
\hline $\begin{array}{l}09: 40- \\
10: 40\end{array}$ & $\begin{array}{l}\text { Session A. Invited Lecture: Antonis Argyros, "Visual Perception } \\
\text { Session Chair: Fillia Makedon }\end{array}$ & of Human Motion and Human-Object Interaction” \\
\hline $\begin{array}{l}10: 40- \\
11: 00\end{array}$ & Coffe & Break (20min) \\
\hline $\begin{array}{l}11: 00- \\
13: 00\end{array}$ & $\begin{array}{l}\text { Session B: Usability and HCI issues } \\
\text { Session Chair: Jaako Hollmen } \\
\text { B1 - Comparing Projected In-Situ Feedback at the Manual } \\
\text { Assembly Workplace with Impaired Workers. Markus Funk, } \\
\text { Andreas Baechler, Liane Baechler, Oliver Korn, Christoph Krieger, } \\
\text { Thomas Heidenreich, Albrecht Schmidt } \\
\text { B2 - SmartTactMaps: A Smartphone-Based Approach to } \\
\text { Support Blind Persons in Exploring Tactile Maps. Timo } \\
\text { Götzelmann, Klaus Winkler } \\
\text { B3 - Pot Hunter: A Virtual Reality Game for Analyzing Range } \\
\text { of Motion. Shawn N. Gieser, Peter Sassaman, Eric Becker, Fillia } \\
\text { Makedon } \\
\text { B4 - About the Development of an Interactive Assistance System } \\
\text { for Impaired Employees in Manual Order Picking. Andreas } \\
\text { Bächler, Peter Kurtz, Thomas Hörz, Georg Krüll, Liane Bächler, Sven } \\
\text { Autenrieth } \\
\text { B5 - Accessible Games for Blind Children, Empowered by } \\
\text { Binaural Sound. Konstantinos Drossos, Nikolaos Zormpas, George } \\
\text { Giannakopoulos, Andreas Floros } \\
\text { B6 - Design Approaches for the Gamification of Production } \\
\text { Environments. A Study Focusing on Acceptance. Oliver Korn, } \\
\text { Markus Funk, Albrecht Schmidt }\end{array}$ & $\begin{array}{l}\text { Session C: Pervasive Systems for the Aged \& Smart Health Devices } \\
\text { Session Chair: Maureen Simmonds } \\
\text { C1 - Test Battery for Assessment of Cognitive Function in Older } \\
\text { Employees: Performance, Brain Processes, and Cardiovascular "costs". } \\
\text { Sergei Schapkin, Xenija Weissbecker-Klaus } \\
\text { C2 - Maintaining Good Relationships in Clinical Setting: the Bonus } \\
\text { DOMUS Project. Kevin Bouchard, Sylvain Giroux, Robert Radziszewski, } \\
\text { Mathieu Gagnon, Quentin Szymanski, Stéphanie Pinard, Mélanie Levasseur, } \\
\text { Nathalie Bier } \\
\text { C3 - Automatic Speed Control for SmartWalker. Jiwon Shin, Ivo Steinmann, } \\
\text { Bertrand Meyer } \\
\text { C4 - A Survey on an Ingestible Sensor for Evaluating Medication } \\
\text { Adherence in Elderly People. Ioannis Kalpouzos, Kostas Giokas, Dimitris } \\
\text { Koutsouris } \\
\text { C5 - Augmenting Everyday Artefacts to Support Social Interaction } \\
\text { Among Senior Peers. Elena Nazzi, Tomas Sokoler } \\
\text { C6 - Cooking Risk Analysis to Enhance Safety of Elderly People in Smart } \\
\text { Kitchen. Rami Yared, Bessam Abdulrazak, Thomas Tessier, Philippe Mabilleau }\end{array}$ \\
\hline $\begin{array}{l}13: 00- \\
14: 00\end{array}$ & \multicolumn{2}{|c|}{ Lunch Break (1 hour) } \\
\hline
\end{tabular}


FIRST DAY: July 1

\begin{tabular}{|c|c|c|}
\hline & Room 1 & Room 2 \\
\hline $\begin{array}{l}14: 00- \\
16: 00\end{array}$ & $\begin{array}{l}\text { Session D. Robotic Devices and Multimodal Interfaces } \\
\text { Session Chair: Taskin Padir } \\
\text { D1 - An Interactive Framework for Learning User-Object } \\
\text { Associations Through Human-Robot Interaction. Michalis } \\
\text { Papakostas, Konstantinos Tsiakas, Natalie Parde, Fillia Makedon, } \\
\text { Vangelis Karkaletsis } \\
\text { D2 - Towards User-Centered Design of a Robotic Prosthetic } \\
\text { Hand with EMG Control Interfaces. Velin Dimitrov, Nicholas Cebry, } \\
\text { Cagdas Onal, Taskin Padir } \\
\text { D3 - Nonverbal Communication With a Humanoid Robot Via } \\
\text { Head Gestures. Salah Saleh, Karsten Berns } \\
\text { D4 - Position Paper: Accessible Human-Robot Interaction } \\
\text { (AHRI). Claudia Loitsch, Michael Schmidt, Gerhard Weber } \\
\text { D5 - Development of MERCURY Version 2.0 Robotic Arms for } \\
\text { Rehabilitation Applications. Nikolaos Moustakas, Panagiotis Kartsidis, } \\
\text { Alkinoos Athanasiou, Alexander Astaras, Panagiotis Bamidis } \\
\text { D6 - Examples for a Ubiquitous Mobility Assistant as Outcome of the } \\
\text { inDAgo Project. Stefanie Müller, Antonija Mrsic Carl, Peter Klein, Henrik Rieß, } \\
\text { Denise Bender (Note: Paper presented in Poster Session) }\end{array}$ & $\begin{array}{l}\text { Session E. Signal \& Image Processing for Ambient Intelligence, Pervasive } \\
\text { Computing } \\
\text { Session Chair: Eric Larson } \\
\text { E1 - Automatic Soundscape Quality Estimation Using Audio Analysis. } \\
\text { Theodoros Giannakopoulos, Georgios Siantikos, Stavros Perantonis, Nefta-Eleftheria } \\
\text { Votsi, John Pantis } \\
\text { E2 - An Interactive and Intuitive STEM Accessibility System for the Blind } \\
\text { and Visually Impaired. Rahul Kumar Namdev, Pattie Maes } \\
\text { E3 - Assistive Positioning System Based Inertial Techniques and Wavelet } \\
\text { Denoising. Teodor Lucian Grigorie, Petre Negrea, Ioana Raluca Edu, Felix } \\
\text { Constantin Adochiei } \\
\text { E4 - An Intra-Fraction Markerless Daily Lung Tumor Localization } \\
\text { algorithm for EPID images. Timothy Rozario, Sergey Bereg, Weihua Mao } \\
\text { E5 - Using Dual Camera Smartphones as Advanced Driver Assistance } \\
\text { Systems: NAVIEYES System Architecture. Mihai Duguleana, Florin Girbacia, } \\
\text { Gheorghe Mogan } \\
\text { E6 - An Integrated RGB-D System for Looking Up the Meaning of Signs. } \\
\text { Christopher Conly, Zhong Zhang, Vassilis Athitsos }\end{array}$ \\
\hline $\begin{array}{l}16: 00- \\
17: 30\end{array}$ & $\begin{array}{r}\text { Poster Session } \\
\text { Also: } \underline{1}^{\text {st }} \text { NSF Docto }\end{array}$ & $\begin{array}{l}\text { mbined with Coffee Break; } \\
\text { Consortium Session (1.5 hours) }\end{array}$ \\
\hline 20:00 & \multicolumn{2}{|c|}{ Welcome Cocktail Party } \\
\hline
\end{tabular}


SECOND DAY: July 2

\begin{tabular}{|c|c|c|}
\hline & Room 1 & Room 2 \\
\hline $\begin{array}{l}08: 40- \\
10: 40\end{array}$ & $\begin{array}{l}\text { Session F. Data Modeling \& Information Management for } \\
\text { Pervasive Assistive Environments } \\
\text { Session Chair: Theodoros Giannakopoulos } \\
\text { F1 - A Framework for Self-managing Database Support and } \\
\text { Parallel Computing for Assistive Systems. Dennis Marten, Andreas } \\
\text { Heuer } \\
\text { F2 - Toward a Platform for Collecting, Mining, and Utilizing } \\
\text { Behavior Data for Detecting Students with Depression Risks. } \\
\text { Einoshin Suzuki, Yutaka Deguchi, Tetsu Matsukawa, Shin Ando, Hiroaki } \\
\text { Ogata, Masanori Sugimoto } \\
\text { F3 - Generating Privacy Constraints for Assistive Environments. } \\
\text { Hannes Grunert, Andreas Heuer } \\
\text { F4 - An Ontological Approach Towards Psychological Patient } \\
\text { Profiling in Pervasive Computing Environments: A case study on } \\
\text { Breast Cancer Patients. Irini Genitsaridi, Manolis Tsiknakis, Kostas } \\
\text { Marias } \\
\text { F5 - Variation in Oxygen Saturation Measurements in Very Low } \\
\text { Birth Weight Infants. Olli-Pekka Rinta-Koski, Jaakko Hollmén, Markus } \\
\text { Leskinen, Sture Andersson }\end{array}$ & $\begin{array}{l}\text { Session G. Tools, Infrastructures, Architectures \& Techniques for } \\
\text { Deploying Pervasive Applications in Assistive Environments } \\
\text { Session Chair: Oliver Korn } \\
\text { G1 - A Prototype of a Real-Time Solution on Mobile Devices for } \\
\text { heart Tele-Auscultation. Julio Cesar Bellido, Giovanna Sannino, Giuseppe } \\
\text { De Pietro } \\
\text { G2 - Sole Based Tactile Information Display for Visually Impaired } \\
\text { Pedestrian Navigation. Wahiba Bouhani, Slim Kammoun, Mohamed Jemni } \\
\text { G3 - Adaptive Music Technology Using the Kinect. Kimberlee Graham- } \\
\text { Knight, George Tzanetakis } \\
\text { G4 - A Multimodal Adaptive Session Manager for Physical } \\
\text { Rehabilitation Exercising. Konstantinos Tsiakas, Manfred Huber, Fillia } \\
\text { Makedon } \\
\text { G5 - Self-Managed Patient-Game Interaction Using the Barrett } \\
\text { WAM Arm for Motion Analysis. Alexandros Lioulemes, Paul Sassaman, } \\
\text { Shawn N. Gieser, Vangelis Karkaletsis, Fillia Makedon, Vangelis Metsis }\end{array}$ \\
\hline $\begin{array}{l}10: 40- \\
10: 55\end{array}$ & \multicolumn{2}{|c|}{ Coffee Break (15 min) } \\
\hline
\end{tabular}


SECOND DAY: July 2

\begin{tabular}{|c|c|c|}
\hline $\begin{array}{l}10: 55 \\
11: 50\end{array}$ & \multicolumn{2}{|c|}{$\begin{array}{l}\text { Session H. Invited Lecture: Vassilis Athitsos, "Computer Vision Methods for Human Motion Analysis" } \\
\text { Session Chair: Panagiotis Papapetrou }\end{array}$} \\
\hline & Room 1 & Room 2 \\
\hline $\begin{array}{l}11: 50- \\
13: 50\end{array}$ & $\begin{array}{l}\text { Session I. Wearable Systems and Monitoring Devices } \\
\text { Session Chair: Vangelis Metsis } \\
\text { I1 - CapWalk: A Capacitive Recognition of Walking-Based Activities } \\
\text { as a Wearable Assistive Technology. Marian Haescher, Denys Matthies, } \\
\text { Gerald Bieber, Bodo Urban } \\
\text { I2 - Human Gait Classification Using a Tri-axial Accelerometer. } \\
\text { Ahmad Lotfi, Minh Nguyen, Caroline Langensiepen } \\
\text { I3 - Sensors Activation Times Prediction in Smart Home. Mohamed } \\
\text { Tarik Moutacalli, Abdenour Bouzouane, Bruno Bouchard } \\
\text { I4 - Performance Characterization of Self-Calibrating Protocols for } \\
\text { Wearable EEG Applications. Thrasyvoulos Karydis, Filipe Aguiar, } \\
\text { Simmie L. Foster, Andreas Mershin } \\
\text { I5 - Reliability Assessment in Everyday-Objects Based Physical- } \\
\text { Activity Sensing Using Personal Information. Claas Richter }\end{array}$ & $\begin{array}{l}\text { Session J. Behavior Modeling, Analysis \& Prediction } \\
\text { Session Chair: George Bebis } \\
\text { J1 - Online Recognition of People's Activities from Raw GPS Data: } \\
\text { Semantic Trajectory Data Analysis. Mehdi Boukhechba, Abdenour } \\
\text { Bouzouane, Bruno Bouchard, Charles Gouin-Vallerand, Sylvain Giroux } \\
\text { J2 - ADOxx Based Tool Support for a Behavior Centered Modeling } \\
\text { Approach. Judith Michael, Fadi Al Machot, Heinrich C. Mayr } \\
\text { J3 - PupilWare: Towards Pervasive Cognitive Load Measurement } \\
\text { Using Commodity Devices. Sohail Rafiqi, Chatchai Wangwiwattana, } \\
\text { Jasmine Kim, Ephrem Fernandez, Suku Nair, Eric Larson } \\
\text { J4 - Predicting Multi-Channel ECGs Using Forests of Randomized } \\
\text { Shapelet Trees. Isak Karlsson, Panagiotis Papapetrou, Lars Asker } \\
\text { J5 - A Multimodal Decision Making System for an Ambient } \\
\text { Assisted Living Environment. Christos Panagiotou, Theodor } \\
\text { Panagiotakopoulos, Achilles Kameas }\end{array}$ \\
\hline $\begin{array}{l}13: 50- \\
14: 50\end{array}$ & \multicolumn{2}{|c|}{ Lunch Break (1 hour) } \\
\hline
\end{tabular}


SECOND DAY: July 2

\begin{tabular}{|c|c|c|}
\hline & Room 1 & Room 2 \\
\hline $\begin{array}{l}14: 30- \\
16: 50\end{array}$ & $\begin{array}{l}\text { Session K. Signal and Image Processing for Ambient Intelligence } \\
\text { \& Pervasive Computing } \\
\text { Session Chair: Sarantos Kapidakis } \\
\text { K1 - A Quantitative Comparison of the Effectiveness of } \\
\text { Instructional Visual Stimuli for Therapy Exercise. Paul Sassaman, } \\
\text { Shawn N. Gieser } \\
\text { K2 - A Survey on Vision-Based Fall Detection. Zhong Zhang, } \\
\text { Christopher Conly, Vassilis Athitsos } \\
\text { K3 - HMAGIC: Head Movement And Gaze Input Cascaded } \\
\text { Pointing. Andrew Kurauchi, Wenxin Feng, Carlos Morimoto, Margrit } \\
\text { Betke } \\
\text { K4 - A Framework for ECG Denoising For Mobile Devices. } \\
\text { Salvatore Cuomo, Ardelio Galletti, Raffaele Farina, Giovanna Sannino, } \\
\text { Giuseppe De Pietro } \\
\text { K5 - Skeleton-Based Human Action Recognition Using Basis } \\
\text { Vectors. Stylianos Asteriadis, Petros Daras } \\
\text { K6 - Thermal Imaging for Active Evacuation Routes. Nikolaos } \\
\text { Doulamis, Anastasios Doulamis, Konstantinos Makantasis, Konstantinos } \\
\text { Karantzalos, Konstantinos Loupos }\end{array}$ & $\begin{array}{l}\text { Session W1. Virtual Reality \& Robotic Technologies for } \\
\text { Rehabilitation Workshop (VRRTR) } \\
\text { Workshop Chair: Stephanie Carey } \\
\text { W11 - Benchmarking Lower Limb Wearable Robots: Emerging } \\
\text { Approaches and Technologies. Diego Torricelli, Antonio J del Ama, Jose } \\
\text { Gonzalez, Juan Moreno, Angel Gil, Jose Pons } \\
\text { W12 - Analysis of Human Stepping Dynamics Using a Wii Balance } \\
\text { Board with a Webcam: A Comparison Study. Ismet Handzic, Kyle Reed } \\
\text { W13 - Eye-Hand Coordination Assessment Method Using a Haptic } \\
\text { Virtual Environment with a Complex Valued Neural Networks } \\
\text { Training Algorithm. Norali Pernalete, Amar Raheja, Alex Knaack } \\
\text { W14 - VR4VR: Vocational Rehabilitation of Individuals With } \\
\text { Disabilities in Immersive Virtual Reality Environments. Lal Bozgeyikli, } \\
\text { Evren Bozgeyikli, Matthew Clevenger, Andrew Raij, Redwan Alqasemi, Rajiv } \\
\text { Dubey } \\
\text { W15 - Development and Evaluation of a Dynamic Virtual Reality } \\
\text { Driving Simulator. Sarah Tudor, Stephanie Carey, Rajiv Dubey } \\
\text { W16 - Simulation Algorithm for the Upper Limb for Better Training } \\
\text { and Prosthesis Prescription for Amputees. Dimitrios Menychtas, } \\
\text { Stephanie Carey, Rajiv Dubey }\end{array}$ \\
\hline $\begin{array}{l}16: 50 \\
17: 50\end{array}$ & \multicolumn{2}{|c|}{$\underline{2^{\text {nd }}}$ NSF Doctoral Consortium Session } \\
\hline 21:00 & \multicolumn{2}{|c|}{ Gala Dinner } \\
\hline
\end{tabular}




\begin{tabular}{|c|c|c|}
\hline & Room 1 & Room 2 \\
\hline $\begin{array}{l}9: 00- \\
11: 00\end{array}$ & $\begin{array}{l}\text { Session L. Tools, Infrastructures, Architectures \& Techniques } \\
\text { for Deploying Pervasive Applications in Assistive Environments } \\
\text { Session Chair: Peter Christian } \\
\text { L1 - Accessing Virtual Classrooms with Assistive Technologies. Carla } \\
\text { Schäkel, Wiebke Köhlmann } \\
\text { L2 - Intelligent Contextual Data Stream Monitoring. Kostas Kolomvatsos, } \\
\text { Christos Anagnostopoulos, Stathes Hadjiefhtymiades } \\
\text { L3 - Cascaded Multimodal Analysis of Alertness Related Features for } \\
\text { Drivers Safety Applications. Mohamed Abouelenien, Mihai Burzo, Rada } \\
\text { Mihalcea } \\
\text { L4 - Instrumented Insole for Weight Measurement of Frail People. Eric } \\
\text { Campo, Yoann Charlon, Damien Brulin } \\
\text { L5 - Wireless Sensor Network Deployment for Remote Elderly Care } \\
\text { Monitoring. Athanasios Dasios, Damianos Gavalas, Grammati Pantziou, } \\
\text { Charalampos Konstantopoulos }\end{array}$ & $\begin{array}{l}\text { Session M. Data Modeling and Information Management for } \\
\text { Pervasive Assistive Environments } \\
\text { Session Chair: Elisabeth Andre } \\
\text { M1 - Empath2: A Flexible Web and Cloud Based Home Health Care } \\
\text { Monitoring System. Robert Dickerson, Enamul Hoque, Ifat Emi, John Stankovic } \\
\text { M2 - Privacy-Preserving Intelligent Networked Video Surveillance for Patient } \\
\text { Monitoring and Alarm Detection. Apostolos Meliones, Simos Kokkovos } \\
\text { M3 - Frequent Pattern Clustering for ADLs Recognition in Smart } \\
\text { Environments. Dany Fortin-Simard, Sebastien Gaboury, Bruno Bouchard, Abdenour } \\
\text { Bouzouane } \\
\text { M4 - Rating Quality in Metadata Harvesting. Sarantos Kapidakis } \\
\text { M5 - Smart Homes and the Challenges of Data. Kevin Bouchard, Sylvain Giroux }\end{array}$ \\
\hline $\begin{array}{l}11: 00- \\
11: 15 \\
\end{array}$ & \multicolumn{2}{|c|}{ Coffee Break (15 min.) } \\
\hline $\begin{array}{l}11: 15- \\
12: 10\end{array}$ & \multicolumn{2}{|c|}{$\begin{array}{l}\text { Session N. Invited Lecture: Elisabeth Andre, "Enhancing Assistive Environments by Empathic Experiences" } \\
\text { Session Chair: Ilias Maglogiannis }\end{array}$} \\
\hline $\begin{array}{l}12: 10- \\
13: 30\end{array}$ & $\begin{array}{l}\text { Session O. Behavior Modeling, Analysis \& Prediction } \\
\text { Session Chair: Dimitrios Zikos } \\
\text { O1 - Modelling and Simulation of Activities of Daily Living } \\
\text { Representing an Older Adult's Behaviour. Abubaker Elbayoudi, Ahmad } \\
\text { Lotfi, Caroline Langensiepen, Kofi Appiah } \\
\text { O2 - Extracting News Text from Web Pages: an Application for the } \\
\text { Visually Impaired. Erik Lundgren, Panagiotis Papapetrou, Lars Asker } \\
\text { O3 - Abnormal Reaching Behaviour in Virtual Environments: } \\
\text { Preliminary Observations. Vaughan Powell, Wendy Powell } \\
\text { O4 - An Automated Framework For Predicting Obstructive Sleep Apnea } \\
\text { Using A Brief, Daytime, Non-Intrusive Test Procedure. Lauren Samy, Paul } \\
\text { Macey, Nabil Alshurafa, Majid Sarrafzadeh }\end{array}$ & $\begin{array}{l}\text { Session W2. RAsEnv -- Workshop on Robotics for Assistive Environments } \\
\text { Workshop Chair: Dan Popa } \\
\text { W21 - A Two-Step Identification Method for Human-Robot Interaction in } \\
\text { Assistive Environments. Alexandros Lioulemes, Nikolaos Sarafianos, Theodoros } \\
\text { Giannakopoulos, Vangelis Karkaletsis } \\
\text { W22 - A Novel EMG-Free Prosthetic Interface System Using Intra-Socket } \\
\text { Force Measurement and Pinch Gestures. Joe Sanford, Oguz Yetkin, Sven Cremer, } \\
\text { Dan Popa } \\
\text { W23 - User Adaptable Tasks for Differential Teaching with Applications to } \\
\text { Robotic Autism Therapy. Isura Ranatunga, Namrata Balakrishnan, Indika } \\
\text { Wijayasinghe, Dan Popa }\end{array}$ \\
\hline
\end{tabular}


THIRD DAY: July 3

\begin{tabular}{|c|c|c|}
\hline $\begin{array}{l}13: 30- \\
14: 30\end{array}$ & \multicolumn{2}{|c|}{ Lunch Break (1 hour) } \\
\hline $\begin{array}{l}14: 30- \\
16: 30\end{array}$ & $\begin{array}{l}\text { Session W3. Non-Invasive Monitoring Technologies for } \\
\text { Disorder Assessment Workshop (SleepMon) } \\
\text { Workshop Chair: Vangelis Metsis } \\
\text { W31 - Monitoring Obstructive Sleep Apnea with Computer- } \\
\text { Aided Diagnosis System Using Electrocardiography and 3-Axis } \\
\text { Acceleration Sensors. Jong-Ha Lee, Eun-Jin Lee, Yoon-Nyun Kim } \\
\text { W32 - Non-Invasive Sleep-Environment Monitoring System. } \\
\text { Fábio Manoel França Lobato, Bruno Almeida da Silva, Rodrigo de Bem, } \\
\text { Diane da Costa Miranda } \\
\text { W33 - Non-Intrusive Infant Monitoring, Sensor Data Fusion } \\
\text { and Tele-Alerting Prototype System (Smart Cot MAIA2). } \\
\text { Miltiadis Yfantis, Lilia Raducan, Alexander Astaras } \\
\text { W34 - Real-Time Subspace Denoising of Polysomnographic } \\
\text { Data. Vangelis Metsis, Ioannis Schizas, Gregg Marshall } \\
\text { W35 - Monitoring Breathing Activity and Sleep Patterns Using } \\
\text { Multimodal Non-Invasive Technologies. Michalis Papakostas, } \\
\text { James Staud, Fillia Makedon, Vangelis Metsis }\end{array}$ & $\begin{array}{l}\text { Session W4. Affective Computing for Biological Activity Recognition in } \\
\text { Assistive Environments Workshop (STHENOS) } \\
\text { Workshop Chair: Ilias Maglogiannis, Dimitris Kosmopoulos } \\
\text { W41 - A Novel Hybrid Approach for Human Silhouette Segmentation. } \\
\text { Konstantinos Delibasis, Theodosis Goudas, Ilias Maglogiannis } \\
\text { W42 - Design of a Lifestyle Recommender System for the Elderly: } \\
\text { Requirement Gatherings in Germany and Greece. Stephan Hammer, Andreas } \\
\text { Seiderer, Elisabeth André, Thomas Rist, Sofia Kastrinaki, Charline Hondrou, } \\
\text { Amaryllis Raouzaiou, Kostas Karpouzis, Stefanos Kollias } \\
\text { W43 - Fall Detection Using History Triple Features. Georgios Goudelis, } \\
\text { Georgios Tsatiris, Konstantinos Karpouzis, Stefanos Kollias } \\
\text { W44 - A Multimodal Adaptive Dialogue Manager for Depressive and } \\
\text { Anxiety Disorder Screening: A Wizard-of-Oz Experiment. Konstantinos } \\
\text { Tsiakas, Lynette Watts, Cyril Lutterodt, Theodoros Giannakopoulos, Alexandros } \\
\text { Papangelis, Robert Gatchel, Vangelis Karkaletsis, Fillia Makedon } \\
\text { W45 - Joint Segmentation and Classification of Actions Using a } \\
\text { Conditional Random Field. Dimitrios Kosmopoulos, Ilias Maglogiannis }\end{array}$ \\
\hline $\begin{array}{l}16: 30- \\
17: 30\end{array}$ & \multicolumn{2}{|c|}{$\begin{array}{l}\text { Special NSF CRI Workshop Combined with Coffee Break } \\
\text { “Developing a Multimodal Human Activity Recognition Repository SMART-MOVE” } \\
\text { Chairs: Fillia Makedon, Vassilis Athitsos } \\
\text { Workshop will be conducted in the form of a panel and discussion open to workshop participants. }\end{array}$} \\
\hline $\begin{array}{l}17: 30- \\
18: 00\end{array}$ & $\begin{array}{r}\text { Conference } \mathbf{C l} \\
\text { Best Technical Paper, Best }\end{array}$ & $\begin{array}{l}\text { sing \& Awards Ceremony } \\
\text { aper for Novelty, and Best Student Paper }\end{array}$ \\
\hline
\end{tabular}




\section{Accepted Posters}

P1. Interpretation of Behaviour Evolution in Activities of Daily Living

Ahmad Lotfi, Caroline Langensiepen, Abubaker Elbayoudi

P2. Exploiting Future Internet Technologies: The Smart Room Case

Charilaos Akasiadis, Evaggelos Spyrou, Georgios Pierris, Dimitris Sgouropoulos, Giorgos Siantikos, Alexandros Mavromatis, Costas Vrakopoulos, Theodoros Giannakopoulos

\section{P3. Flexible Metadata Mapping Using OAI-PMH}

Sarantos Kapidakis, Nikos Houssos, Panagiotis Koutsourakis, Kostas Stamatis

P4. ifONLY - A Smart Phone App to Capture Unmet Need and Support the Development of New Products for People Living with Long- Term Conditions

Lisa Austin, Nigel Harris, Christine Sidenko

P5. Development and Evaluation of a Unity-based, Kinect-controlled Avatar for Physical Rehabilitation Dylan Ebert, Vangelis Metsis, Fillia Makedon

P6. A Novel, Non-Invasive Dermatological Diagnostic Instrument for Skin Resistance Scanning (DermaSense) Inessa Kirsanidou, Alexander Zogkas, Christina Kemanetzi, Chrysovalantis Korfitis, Elizabeth Lazaridou, Alexander Astaras

P7. Monitoring of Compliance on an Individual Treatment Through Mobile Innovations

Athanasios Anastasiou, Kostas Giokas, Dimitra Iliopoulou, Dimitris Koutsouris

P8. Mobile-Cloud Platform for Raising Hearing Loss Awareness

Panagiotis Katrakazas, Kostas Giokas, Dimitra Iliopoulou, Dimitris Koutsouris

P9. Exploring Assistive Technology for Assistance Dog Owners in Emergency Situations

Charlotte Robinson, Clara Mancini, Janet van der Linden, Lydia Swanson, Claire Guest

P10. A Framework for the Assessment of Wandering Behavior

Theodora Toutounzi, Scott Phan, Fillia Makedon

P11. Large Scale Ebola Detection

Shyuan Yang, Barry Fine, Ioannis Kymissis

P12. 3D Mapping of Visual Attention for Smart Rehabilitation

Christopher McMurrough, Alexandros Lioulemes, Scott Phan, Fillia Makedon

P13. Self-Calibrating Protocols Enhance Wearable EEG Diagnostics and Consumer Applications

Thrasyvoulos Karydis, Filipe Aguiar, Simmie Foster, Andreas Mershin

P14. Robot-Aided Rehabilitation using Force Analysis

Maher Abujelala, Alexandros Lioulemes, Paul Sassaman, Fillia Makedon

P15. RF Powered Sleep Apnea Monitoring System

Vishnoukumaar Sivaji, Dinesh Bhatia, Shalini Prasad

P16. An Online Learning Approach for Trend Recognition

David Paulk

P17. Using Binary Logistic Regression Coefficients for the Dynamic Quantification of Comorbidities

Dimitrios Zikos, Ismail Vandeliwala 


\section{Program Committee}

Ilias Maglogiannis (Co-Chair) - Univ. of Piraeus, Greece

Vangelis Metsis (Co-Chair) - Texas State University, USA

Gian-Luca Mariottini (Co-Chair) - Univ. of Texas at Arlington, USA

Oliver Korn (Co-Chair) - Korion Simulation \& Assistive Technology GmbH, Germany

Dimitra Anastasiou - Univ. of Bremen, German

Antonis Argyros - ICS-FORTH, Greece

Alexander Astaras - American College of Thessaloniki, Greece

Vassilis Athitsos - Univ. of Texas at Arlington, USA

Lynne Baillie - Glasgow Caledonian University, UK

Panagiotis Bamidis - Aristotle Univ. of Thessaloniki, Greece

Eric Becker - Univ. of Texas at Arlington, USA

Dinesh K. Bhatia - Univ. of Texas at Dallas, USA

Gerald Bieber - Fraunhofer Institute for Computer Graphics Research IGD, Germany

Matt Bishop - Univ. of California at Davis, USA

Paolo Bonato - Wyss Institute at Harvard Univ., USA

Matt-Mouley Bouamrane - Univ. of Glasgow, UK

Stephanie L. Carey - CARRT; Univ. of South Florida, USA

Christina Choi - Georgia Institute of Technology, USA

Paul Doliotis - Wynright Robotics, USA

Xiaohu Guo - Univ. of Texas at Dallas, USA

Jaakko Hollmen - Aalto Univ., Finland

Junzhou Huang - Univ. of Texas at Arlington, USA

Michael Justiss - Indiana Univ., USA

Ioannis Kakadiaris - Univ. of Houston, USA

Sarantos Kapidakis - Ionian Univ., Greece

Vangelis Karkaletsis - NCSR Demokritos, Greece

Dimitrios Kosmopoulos - Univ. of Patras; ICS-FORTH, Greece

Marilyn Lennon - Univ. of Strathclyde, UK

Fillia Makedon - Univ. of Texas at Arlington, USA

Christopher McMurrough - Univ. of Texas at Arlington, USA

Taskin Padir - Worcester Polytechnic Institute, USA

Panagiotis Papapetrou - Stockholm Univ., Sweden

Lorna Paul - Univ. of Glaskow, UK

Christian Peter - BioArtProducts GmbH, Germany

Dan Popa - Univ. of Texas at Arlington, USA

Majid Sarrafzadeh - Univ. of California, Los Angeles, USA

Sergei Schapkin - Federal Institute for Occupational Safety and Health, Germany

Li Shen - Indiana Univ., USA

Maureen J. Simmonds - Univ. of Texas Health Science Center at San Antonio, USA

Isabelle Viaud-Delmon - IRCAM, France

Maria Wolters - Univ. of Edinburgh, UK

Xenophon Zabulis - ICS-FORTH, Greece

Dimitrios Zikos - Univ. of Texas at Arlington, USA 
Conference Proceedings Editorial Committee

Konstantinos Tsiakas - Univ. of Texas at Arlington USA

Michalis Papakostas - Univ. of Texas at Arlington USA

Shawn Gieser - Univ. of Texas at Arlington USA

Dimitrios Zikos - Univ. of Texas at Arlington USA

Scott Phan - Univ. of Texas at Arlington USA

\section{Workshops Chairs}

Dimitrios Kosmopoulos - TEI of Crete and Institute of Computer Science, FORTH, Greece

Vangelis Metsis - Univ. of Texas at Arlington, USA

Ilias Maglogiannis - Univ. of Piraeus, Greece

Dan Popa - Univ. of Texas at Arlington, USA

Stephanie L. Carey-CARRT; Univ. of South Florida, USA

\section{Organizing and Technical Arrangements Committee [petrae@uta.edu]}

Fillia Makedon (Conference Chair) - Univ. of Texas at Arlington, USA

Scott Phan - Univ. of Texas at Arlington, USA

Alexandros Lioulemes - Univ. of Texas at Arlington, USA

Michalis Papakostas - Univ. of Texas at Arlington, USA

Shawn Gieser - Univ. of Texas at Arlington, USA

Maher Abujelala - Univ. of Texas at Arlington, USA

Theodora Toutountzi - Univ. of Texas at Arlington, USA

Konstantinos Tsiakas - Univ. of Texas at Arlington, USA

Cyril Lutterodt - Univ. of Texas at Arlington, USA

Zhong Zhang - Univ. of Texas at Arlington, USA

\section{NSF Doctoral Consortium (DC) Students}

1. Velin Dimitrov - Worchester Polytechnic Institute

2. Vishnou Kumaar - Univ. of Texas at Dallas

3. Nikolaos Sarafianos - Univ. of Houston

4. Shyuan Yang-Colombia Univ.

5. Shawn Gieser - Univ. of Texas at Arlington

6. Thrasyvoulos Karydis - Massachusetts Institute of Technology

7. Rahul Namdev - Massachusetts Institute of Technology

8. Timothy Rozario - Univ. of Texas at Dallas

9. Ifat Emi - Univ. of Virginia

10. Dimitrios Menychtas - Univ. South Florida

11. Lauren Samy - Univ. of California at Los Angeles

12. Andrew Kurauchi - Boston Univ.

13. Chris Conly - Univ. of Texas at Arlington

The following additional DC students were covered by UTA MATCHING funds:

14. Konstantinos Tsiakas - Univ. of Texas at Arlington

15. Michalis Papakostas - Univ. of Texas at Arlington

16. Alexandros Lioulemes - Univ. of Texas at Arlington

17. Oguz Yetkin-Univ. of Texas at Arlington

18. David Paulk - Undergraduate - Princeton Univ.

19. James Staud - Undergraduate - Univ. of Texas at Arlington

20. Dylan Ebert - Undergraduate - Univ. of Texas at Arlington

21. Caleb Gentry - Undergraduate - Univ. of Texas at Arlington

22. Raith Hamzah - Undergraduate - Univ. of Texas at Arlington

\section{NSF Doctoral Consortium Faculty}

Fillia Makedon (Chair) - Univ. of Texas at Arlington, USA

Vangelis Metsis (Co-Chair) - Texas State Univ., USA

Vassilis Athitsos - Univ. of Texas at Arlington, USA

Alexandra Stefan - Univ. of Texas at Arlington, USA

Dimitrios Zikos - Univ. of Texas at Arlington, USA 